\title{
Collective modes in a two-band superfluid of ultracold alkaline-earth-metal atoms close to an orbital Feshbach resonance
}

\author{
Yi-Cai Zhang, Shanshan Ding, and Shizhong Zhang \\ Department of Physics and Center of Theoretical and Computational Physics, The University of Hong Kong, Hong Kong, China
}

(Received 6 July 2016; published 27 April 2017)

\begin{abstract}
We discuss the collective modes in an alkaline-earth-metal Fermi gas close to an orbital Feshbach resonance. Unlike the usual Feshbach resonance, the orbital Feshbach resonance in alkaline-earth-metal atoms realizes a two-band superfluid system where the fermionic nature of both the open and the closed channel has to be taken into account. We show that, apart from the usual Anderson-Bogoliubov mode which corresponds to the oscillation of total density, there also appears the long-sought Leggett mode corresponding to the oscillation of relative density between the two channels. The existence of the phonon and the Leggett modes and their evolution are discussed in detail. We show how these collective modes are reflected in the density response of the system.
\end{abstract}

DOI: 10.1103/PhysRevA.95.041603

Introduction. A recent theoretical proposal [1] and the subsequent experimental confirmations [2,3] of the so-called orbital Feshbach resonance (OFR) in alkaline-earth-metal atoms have opened a new avenue to investigate strongly interacting Fermi gases. A prominent feature of the alkalineearth-metal atoms, such as $\mathrm{Sr}$ [4] and $\mathrm{Yb}$ [5-7], is that there are two clock states, the electronic $s$ and $p$ states, both of which have zero electronic angular momentum $J=0$. As a result, there is no hyperfine coupling between the electronic and nuclear spins. The interatomic interactions which depend (primarily) on the electronic configurations thus become independent of nuclear spins. This realizes the so-called $\mathrm{SU}(N)$ symmetries, where $N$ is the number of nuclear spin components [8-16]. The OFR relies on the atoms residing on both the $s$ and $p$ states which possess slightly different Landé $g$ factors $[17,18]$, thus allowing tuning by an external magnetic field. The enlarged symmetries with $N>2$ are also quite relevant to other fields of physics, for example, in quantum chromodynamics, where $N=3$ describes either the color or flavor group [19] and $N=6$ in nuclear physics [20].

Unlike the usual Feshbach resonance, where the closedchannel bound state responsible for resonance has a binding energy relative to its scattering threshold that is much larger than the Fermi energy of the system, OFR in turn relies on the existence of a shallow two-body bound state whose binding energy is of the order of the Fermi energy, and thus brings in new features that have not been encountered before. In particular, it is no longer admissible to treat the closed-channel state as a single boson without taking into account its proper internal dynamics. One is thus led to a situation that is much the same as a two-band superconductor, where the appropriate picture is that two Fermi surfaces (including both spin) intersect the chemical potential [21-23]. We show that this new situation leads to the appearance of new collective modes and, in particular, the long-sought Leggett mode in the two-band superconductor. The dependences of the collective modes on the tuning parameters are analyzed in detail. We also calculate the dynamic structure factor of the system and identify the appearance of the Leggett mode in the oscillations of the relative density.

The model Hamiltonian. Let us denote the electronic ground $s$ state as $g$ and the excited $p$ state as $e$. Then the Hamiltonian of alkaline-earth-metal fermions close to an orbital Feshbach resonance is given by $H=H_{\mathrm{o}}+H_{\mathrm{c}}+H_{\text {int }}$ [1], where (setting $m=\hbar=1$ )

$$
\begin{gathered}
H_{\mathrm{o}}=\sum_{\mathbf{k}} \epsilon_{\mathbf{k}}\left(c_{g \downarrow, \mathbf{k}}^{\dagger} c_{g \downarrow, \mathbf{k}}+c_{e \uparrow, \mathbf{k}}^{\dagger} c_{e \uparrow, \mathbf{k}}\right), \\
H_{\mathrm{c}}=\sum_{\mathbf{k}}\left(\epsilon_{\mathbf{k}}+\delta / 2\right)\left(c_{g \uparrow, \mathbf{k}}^{\dagger} c_{g \uparrow, \mathbf{k}}+c_{e \downarrow, \mathbf{k}}^{\dagger} c_{e \downarrow, \mathbf{k}}\right), \\
H_{\mathrm{int}}=-\frac{g_{+}}{2} A_{+}^{\dagger} A_{+}-\frac{g_{-}}{2} A_{-}^{\dagger} A_{-} .
\end{gathered}
$$

Here, $H_{\mathrm{o}}\left(H_{\mathrm{c}}\right)$ is the single-particle Hamiltonian for the open (closed) channel, $\epsilon_{\mathbf{k}}=k^{2} / 2-\mu$, with $\mu$ being the chemical potential. $\delta$ is the detuning between the open and closed channels and can be tuned by an external magnetic field. $A_{+}=\sum_{\mathbf{k}}\left(c_{e \downarrow, \mathbf{k}} c_{g \uparrow,-\mathbf{k}}-c_{e \uparrow, \mathbf{k}} c_{g \downarrow,-\mathbf{k}}\right)$ and $A_{-}=$ $\sum_{\mathbf{k}}\left(c_{e \downarrow, \mathbf{k}} c_{g \uparrow,-\mathbf{k}}+c_{e \uparrow, \mathbf{k}} c_{g \downarrow,-\mathbf{k}}\right)$. Here, $g_{+(-)}>0$ are the coupling constants indicating the strength of the attractive interaction for the singlet (triplet) orbital channel and related to the respective scattering lengths $a_{+/-}$by the usual renormalization conditions, $1 / g_{+/-}=-1 / 4 \pi a_{+/-}+\sum_{\mathbf{k}} 1 / k^{2}$.

To investigate the pairing structure of Fermi gases close to OFR, we define the order parameters $\Delta_{+/-} \equiv$ $-g_{+/-}\left\langle A_{+/-}\right\rangle / 2$, or, more conveniently, $\Delta_{\mathrm{o}} \equiv \Delta_{-}-\Delta_{+}$ and $\Delta_{\mathrm{c}} \equiv \Delta_{-}+\Delta_{+}$for the pairing strengths in the open and closed channels, respectively. Introducing the Grassman variable in the Nambu notation, $\bar{\Psi}=\left(\bar{\psi}_{g \downarrow}, \psi_{e \uparrow}, \bar{\psi}_{g \uparrow}, \psi_{e \downarrow}\right)$, the partition function of the system can be written as, after a Hubbard-Stratonovich transformation, $\mathcal{Z} \equiv e^{-\beta \Omega}=$ $\int D \bar{\Psi} D \Psi D \bar{\Delta}_{\mathrm{o}} D \Delta_{\mathrm{o}} D \bar{\Delta}_{\mathrm{c}} D \Delta_{\mathrm{c}} \exp (-S)$, where the effective action

$$
S=-\int d x\left[\bar{\Psi} G^{-1} \Psi-\frac{2\left|\Delta_{+}(x)\right|^{2}}{g_{+}}-\frac{2\left|\Delta_{-}(x)\right|^{2}}{g_{-}}\right],
$$

where $x=\{\tau, \vec{r}\}, \int d x \equiv \int_{0}^{\beta} d \tau \int d^{3} \vec{r}$, with $\beta=1 / k_{\mathrm{B}} T$ being the inverse temperature and $\Omega$ is the thermodynamical potential. In this Rapid Communication, we focus on the zero-temperature physics and take the limit of $\beta \rightarrow \infty$ at the end of the calculation. The inverse Green's function $G^{-1}$ is given by

$$
G^{-1}\left(x, x^{\prime}\right)=\delta\left(x-x^{\prime}\right)\left(\begin{array}{cc}
G_{\mathrm{o}}^{-1} & 0 \\
0 & G_{\mathrm{c}}^{-1}
\end{array}\right)
$$




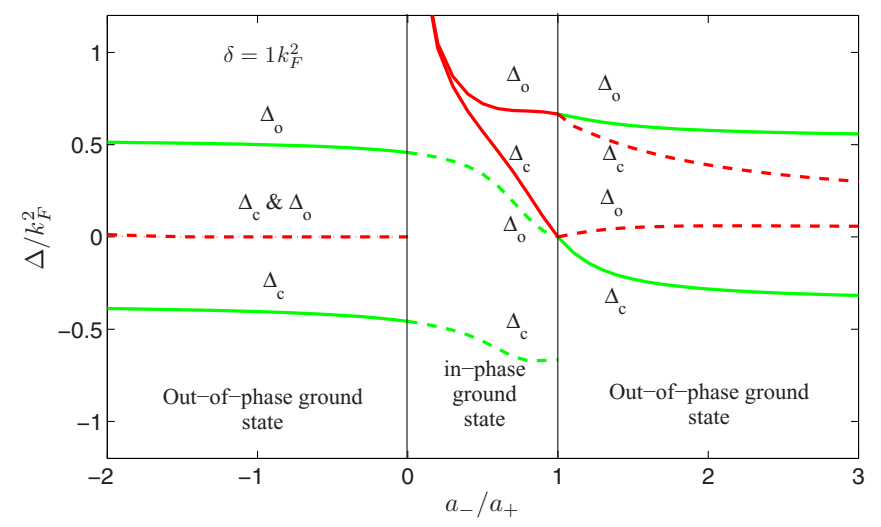

FIG. 1. The order parameters for the two-band superfluid close to an orbital Feshbach resonance. Depending on the values of $a_{-} / a_{+}$, there are in general two types of solutions to the mean-field equation. The red lines indicate an in-phase solution while the green lines are for the out-of-phase solution. The solid lines indicate a stable solution and the dashed lines are for the saddle-point solution for a specific parameter range of $a_{-} / a_{+}$. We choose the parameters $\delta=k_{F}^{2}$.

with the matrix Green's functions $G_{\mathrm{o}, \mathrm{c}}^{-1}$ given by

$$
G_{\mathrm{o}}^{-1}(x)=\left(\begin{array}{cc}
-\partial_{\tau}+\nabla^{2} / 2+\mu & \Delta_{\mathrm{o}}(x) \\
\Delta_{\mathrm{o}}^{*}(x) & -\partial_{\tau}-\nabla^{2} / 2-\mu
\end{array}\right)
$$

and

$$
G_{\mathrm{c}}^{-1}(x)=\left(\begin{array}{cc}
-\partial_{\tau}+\frac{\nabla^{2}}{2}-\frac{\delta}{2}+\mu & \Delta_{\mathrm{c}}(x) \\
\Delta_{\mathrm{c}}^{*}(x) & -\partial_{\tau}-\frac{\nabla^{2}}{2}+\frac{\delta}{2}-\mu
\end{array}\right) .
$$

Integrating over the fermionic field, one finds the effective action $S_{\Delta}$ in terms of $\Delta_{\mathrm{o}, \mathrm{c}}$, given by

$$
S_{\Delta}=\int d x\left[\frac{2\left|\Delta_{+}(x)\right|^{2}}{g_{+}}+\frac{2\left|\Delta_{-}(x)\right|^{2}}{g_{-}}\right]-\operatorname{Tr} \ln G^{-1}\left(x, x^{\prime}\right) .
$$

In the following, we choose the parameter such that $k_{F} a_{+}=1$, and investigate the evolution of the collective mode as one changes $a_{-}$. Here, $k_{F}$ is determined by the density through $n=k_{F}^{3} /\left(3 \pi^{2}\right)$.

Mean-field solution and its stability. Within the mean-field approximation, the optimal values of the order parameters $\Delta_{\mathrm{o}, \mathrm{c}}$ are given by the saddle-point equations: $\partial \Omega / \partial \Delta_{\mathrm{o}, \mathrm{c}}=0$. On the other hand, the chemical potential is determined by $\partial \Omega / \partial \mu=-n$, with $n$ the average density. For more details, see the Supplemental Material [24]. It turns out that, depending on the value of $a_{-} / a_{+}$, there are two types of solutions: the true ground state and the metastable state. For $0<a_{-} / a_{+}<1$, the ground state corresponds to the case when two order parameters are in phase, $\Delta_{\mathrm{c}} \Delta_{\mathrm{o}}>0$, while the out-of-phase solution $\Delta_{\mathrm{c}} \Delta_{\mathrm{o}}<0$ is the metastable state. On the other hand, for $a_{-} / a_{+}<0$ or $a_{-} / a_{+}>1$, the out-of-phase solution is the true ground state, while the in-phase solution is the metastable solution; see Fig. 1.

To understand the various mean-field solutions, we assume that $a_{+}>0$. The coupling energy between the two channels within the mean-field approximation is given by $\left\langle V_{\mathrm{oc}}\right\rangle=$ $2\left(1 / a_{+}-1 / a_{-}\right)\left|\Delta_{\mathrm{o}} \Delta_{\mathrm{c}}\right| \cos \left(\theta_{\mathrm{o}}-\theta_{\mathrm{c}}\right)$, where $\theta_{\mathrm{o}, \mathrm{c}}$ are the phases of the order parameters $\Delta_{\mathrm{o}, \mathrm{c}}$. When $0<a_{-}<a_{+}$, the bound state in the triplet potential (determined by $a_{-}$) has lower energy and, as a result, the triplet pairing order parameter $\Delta_{-}=\Delta_{\mathrm{o}}+\Delta_{\mathrm{c}}$ is dominant over the singlet component $\Delta_{+}$. As a result, in order to minimize the coupling energy $\left\langle V_{\mathrm{oc}}\right\rangle$, $\Delta_{\mathrm{o}} \Delta_{\mathrm{c}}>0$ and the in-phase solution is the ground state. On the other hand, when $a_{-}>a_{+}$or $a_{-}<0$, the singlet channel is the dominant pairing component and the out-of-phase solution $\Delta_{\mathrm{o}} \Delta_{\mathrm{c}}<0$ is the true ground state. Hence, it is expected that as one changes $a_{-}$, the ground-state properties will change from a singlet dominant pairing to a triplet one. At the transition when $a_{-}=a_{+}$, the open and closed channels decouple (the coupling constant $\propto a_{-}-a_{+}$) [1]. The two channels become independent from each other, so the the out-of-phase and in-phase solutions are degenerate; see Fig. 1. The experimental interaction parameters near the orbital Feshbach resonance of ${ }^{173} \mathrm{Yb}$ atomic gas are $a_{+} \sim 1900 a_{0}$ and $a_{-} \sim 200 a_{0}$, giving $a_{-} / a_{+} \sim 0.1[2,3]$. So the ground state of the system is an in-phase solution of $\Delta_{c} \Delta_{0}>0$ close to OFR. Here, we shall mainly focus on the true ground state and its associated collective excitations for different parameters of $a_{-} / a_{+}$and detuning $\delta$. It is to be emphasized that while the mean-field description close to resonance captures the qualitative behavior of the system, the precise values of the gap parameters require exact numerical calculations.

Collective excitations. To investigate the fluctuation around the mean-field solutions, we write $\Delta_{\mathrm{o}, \mathrm{c}}(x)=\Delta_{\mathrm{o}, \mathrm{c}}+\eta_{\mathrm{o}, \mathrm{c}}(x)$. Transforming to the momentum and Matsubara frequency space, we can write the inverse Green's function as $G^{-1}\left(k, k^{\prime}\right)=G_{0}^{-1}(k) \delta\left(k-k^{\prime}\right)+K\left(k, k^{\prime}\right)$, where

$$
G_{0}^{-1}(k)=\left(\begin{array}{cccc}
i \omega_{n}-\epsilon_{k}^{\mathrm{o}} & \Delta_{o} & 0 & 0 \\
\Delta_{o}^{*} & i \omega_{n}+\epsilon_{k}^{\mathrm{o}} & 0 & 0 \\
0 & 0 & i \omega_{n}-\epsilon_{k}^{\mathrm{c}} & \Delta_{c} \\
0 & 0 & \Delta_{c}^{*} & i \omega_{n}+\epsilon_{k}^{\mathrm{c}}
\end{array}\right),
$$

and the matrix $K\left(k, k^{\prime}\right)$ is given by

$$
K\left(k, k^{\prime}\right)=\left(\begin{array}{cccc}
0 & \eta_{\mathrm{o}}(-q) & 0 & 0 \\
\eta_{\mathrm{o}}^{*}(q) & 0 & 0 & 0 \\
0 & 0 & 0 & \eta_{\mathrm{c}}(-q) \\
0 & 0 & \eta_{\mathrm{c}}^{*}(q) & 0
\end{array}\right) .
$$

Here, $\quad k=\left\{i \omega_{n}, \mathbf{k}\right\} \quad$ and $\quad \delta\left(k-k^{\prime}\right) \equiv \delta^{3}\left(\mathbf{k}-\mathbf{k}^{\prime}\right) \delta_{n n^{\prime}} . \quad \epsilon_{\mathbf{k}}^{\mathrm{o}}=$ $k^{2} / 2-\mu$ and $\epsilon_{\mathbf{k}}^{\mathbf{c}}=k^{2} / 2+\delta / 2-\mu$ are the kinetic energies of the open and closed channels measured from the chemical potential $\mu$. The four-momentum transfer $q=k-k^{\prime} \equiv\{\mathbf{k}-$ $\left.\mathbf{k}^{\prime}, i \omega_{n}-i \omega_{n^{\prime}}\right\}$. The fluctuation contribution to the effective action then can be written in the usual quadratic form $S_{\eta}=$ $\frac{1}{2} \sum_{q} \bar{\eta}_{q} M(q) \eta_{q}$, where the fluctuation matrix is given by [25]

$$
M=\left(\begin{array}{cccc}
M_{11}^{\mathrm{o}}(q) & M_{12}^{\mathrm{o}}(q) & d g & 0 \\
M_{21}^{\mathrm{o}}(q) & M_{22}^{\mathrm{o}}(q) & 0 & d g \\
d g & 0 & M_{11}^{\mathrm{c}}(q) & M_{12}^{\mathrm{c}}(q) \\
0 & d g & M_{21}^{\mathrm{c}}(q) & M_{22}^{\mathrm{c}}(q)
\end{array}\right) .
$$


(a)
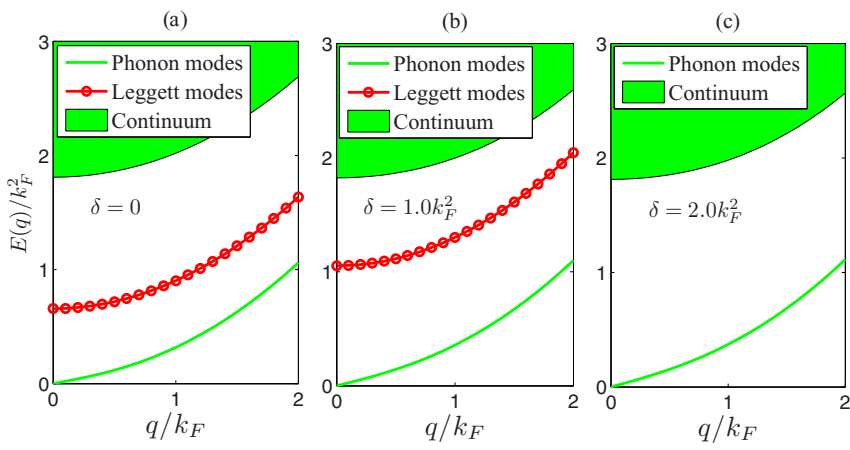

FIG. 2. The dispersions of the collective excitations for different detunings for the ground state with $a_{-} / a_{+}=0.8$. (a) For $\delta=0$, both Leggett and phonon modes are well defined and below the quasiparticle continuum. (b) As detuning increased to $\delta=1 k_{F}^{2}$, the gap for the Leggett mode at $\mathbf{q}=\mathbf{0}$ is pushed upwards. (c) For $\delta=2 k_{F}^{2}$, only the phonon mode remains and the Leggett mode merges into the continuum.

Here, $\quad \bar{\eta}_{q}=\left[\eta_{\mathrm{o}}^{*}(q), \eta_{\mathrm{o}}(-q), \eta_{\mathrm{c}}^{*}(q), \eta_{\mathrm{c}}(-q)\right] \quad$ and $\quad d g=$ $1 / 2\left(1 / g_{-}-1 / g_{+}\right)=-1 / 8 \pi\left(1 / a_{-}-1 / a_{+}\right)$. The various $M$ 's are given by

$$
\begin{gathered}
M_{11}^{\mathrm{o}}(q)=\frac{1}{\beta} \sum_{k} G_{\mathrm{o} 11}(k+q) G_{\mathrm{o} 22}(k)+\frac{1}{2}\left[\frac{1}{g_{+}}+\frac{1}{g_{-}}\right] \\
M_{12}^{\mathrm{o}}(q)=\frac{1}{\beta} \sum_{k, n^{\prime}} G_{\mathrm{o} 12}(k+q) G_{\mathrm{o} 12}(k) \\
M_{21}^{\mathrm{o}}(q)=M_{12}^{\mathrm{o}}(q) \\
M_{22}^{\mathrm{o}}(q)=M_{11}^{\mathrm{o}}(-q)
\end{gathered}
$$

and similarly for the closed channel. The collective modes are given by the zeros of determinant $\operatorname{Det}\left|M\left(\mathbf{q}, \omega+i 0^{+}\right)\right|=$ 0 [26].

In Figs. 2(a)-2(c), we show the excitation spectra for three different detunings $\delta=0,1,2$ (in units of $k_{F}^{2}$ ) and for fixed values of $a_{-} / a_{+}=0.8$. Because of the existence of two bands, in addition to the usual Goldstone (or Anderson-Bogoliubov) modes (green solid lines in Fig. 2), which correspond to the oscillation of total density, an additional Leggett mode appears (red solid lines with circles), which corresponds to the oscillation of the relative densities of the two bands [27]. With increasing detuning $\delta$, the Leggett modes gradually merge into the two quasiparticle continua and are heavily damped.

One can understand the appearance of the Leggett mode as follows. In the absence of interchannel coupling $\left(a_{-}=a_{+}\right)$, the pairing occurs in the closed and the open channel independently. As a result, two phonon modes appear corresponding to the independent density fluctuations in the two channels. As the interchannel coupling $\left(a_{-} \neq a_{+}\right)$is turned on, there still remains a phonon mode corresponding to the total density fluctuations, while the fluctuation of the relative densities in the two channels acquires a nonzero gap due to interchannel coupling. In Fig. 3(b), we calculated the variations of the gap of the Leggett mode $\omega_{L}(\mathbf{q}=\mathbf{0})$ with the scattering length $a_{-} / a_{+}$in the ground states. As expected, near the phase
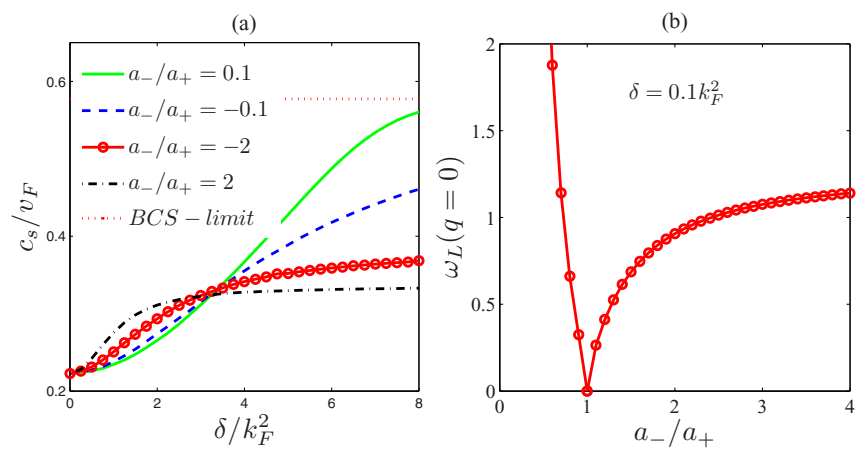

FIG. 3. (a) The evolution of sound velocity $c_{s}$ with detuning $\delta$ for various parameters $a_{-} / a_{+}$for the out-of-phase solution $\Delta_{0} \Delta_{c}<0$. The dotted line indicates the limiting value with $c_{s}=1 / \sqrt{3} v_{F}$ in the BCS limit. (b) The evolution of the gap for the Leggett mode with $a_{-} / a_{+}$for $\delta=0.1 k_{F}^{2}$ in the ground states. Note that the gap closes at $a_{-} / a_{+}=1$ where the coupling between the two channels vanishes.

transition point $\left(a_{-} / a_{+}=1\right)$ where interchannel coupling vanishes, $\omega_{L}(\mathbf{q}=\mathbf{0}) \rightarrow 0$.

Figure 3(a) shows the variations of sound velocity as a function of $\delta$ with different $a_{-} / a_{+}$for an out-of-phase solution $\left(\Delta_{\mathrm{o}} \Delta_{\mathrm{c}}<0\right)$. When the detuning $0<a_{-} / a_{+}<1$, the system is metastable (a saddle point of energy), however, there still exist well-defined phonon modes (green solid line) in Fig. 3(a). This is because, for $q \rightarrow 0$, the phonon modes correspond to the in-phase density oscillation of two channels that is along the direction in which the energy increases. In addition, with increasing $\delta$, the system enters into the BCS limit [1]. Consequently, the sound velocity also saturates to its BCS limit value $c_{s}=v_{F} / \sqrt{3}$ (red dotted line) [25]. For several other values of $a_{-} / a_{+}=-0.1,-2,2$, the system is in its true ground state and the sound velocity increases with am increase of detuning $\delta$. When $\delta=0$, the Hamiltonian can be written as two independent (singlet and triplet orbital) channel Hamiltonians by reorganizing the single-particle Hamiltonian of Eq. (1). The out-of-phase solution would satisfy $\Delta_{o}=-\Delta_{\mathrm{c}}$. Hence only the singlet orbital pairing $\Delta_{+}=\left(\Delta_{\mathrm{c}}-\Delta_{\mathrm{o}}\right) / 2$ occurs, while the triplet orbital pairing $\Delta_{-}=\left(\Delta_{\mathrm{c}}+\Delta_{\mathrm{o}}\right) / 2$ vanishes. Consequently, the thermodynamical potential does not depend on $a_{-}$and the sound velocities for different $a_{-} / a_{+}$ are same. In addition, for fixed $\delta$, the sound velocity increases when $a_{-} / a_{+}$varies from -0.1 to 2 when detuning is small while it deceases for larger $\delta$.

Dynamical structure factor. As a direct probe of the Leggett mode, we can make use of Bragg spectroscopy [28]. Let us define the density correlation function matrix as [24]

$$
\chi(q) \equiv-\left(\begin{array}{ll}
\left\langle\delta \rho_{\mathrm{o}}(-q) \delta \rho_{\mathrm{o}}(q)\right\rangle & \left\langle\delta \rho_{\mathrm{o}}(-q) \delta \rho_{\mathrm{c}}(q)\right\rangle \\
\left\langle\delta \rho_{\mathrm{c}}(-q) \delta \rho_{\mathrm{o}}(q)\right\rangle & \left\langle\delta \rho_{\mathrm{c}}(-q) \delta \rho_{\mathrm{c}}(q)\right\rangle
\end{array}\right),
$$

where $\delta \rho_{\mathrm{o}, \mathrm{c}}(q)$ are the density fluctuation operators in the open and closed channels. The detailed form of $\chi(q)$ is given in the Supplemental Material [24]. The dynamical structure factor for the total density fluctuation, $\rho_{\mathbf{q}}=\rho_{\mathbf{q}}^{\mathbf{o}}+\rho_{\mathbf{q}}^{\mathbf{c}}$, is given by $\left(i \omega_{n} \rightarrow \omega+0^{+}\right)$

$$
S_{\text {total }}(\mathbf{q}, \omega)=-\frac{1}{\pi} \operatorname{Im}\left(\chi_{11}+\chi_{12}+\chi_{21}+\chi_{22}\right),
$$


(a)

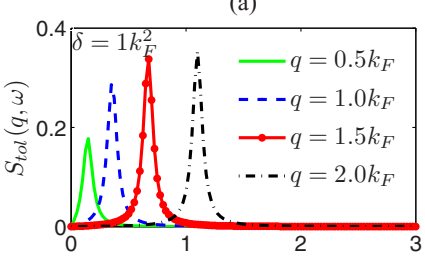

(c)

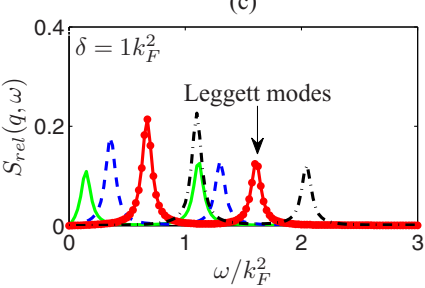

(b)

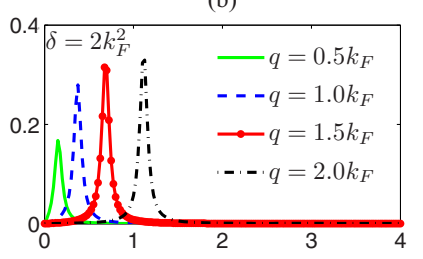

(d)

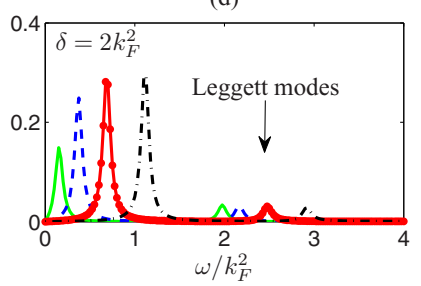

FIG. 4. The dynamical structure factors for (a), (b) the total density and (c), (d) the relative density for $\delta=1 k_{F}^{2}$ and $\delta=2 k_{F}^{2}$ with $a_{-} / a_{+}=0.8$. (a), (b) Only the phonon mode is present in $S_{\text {total }}(\mathbf{q}, \omega)$ and its frequency increases with wave vector $q$. (c), (d) $S_{\text {rel }}(\mathbf{q}, \omega)$ features peaks corresponding to the Leggett mode at relative higher energies.

and that for the relative density $\left(m_{\mathbf{q}} \equiv \rho_{\mathbf{q}}^{\mathrm{o}}-\rho_{\mathbf{q}}^{\mathrm{c}}\right)$ oscillation is given by

$$
S_{\text {rel }}(\mathbf{q}, \omega)=-\frac{1}{\pi} \operatorname{Im}\left(\chi_{11}-\chi_{12}-\chi_{21}+\chi_{22}\right) .
$$

The dynamical structure factor $S_{\text {total }}$ satisfies the famous $f$-sum rule $\int d \omega \omega S_{\text {total }}(\mathbf{q}, \omega)=\left[\left[\rho_{\mathbf{q}}, H\right], \rho_{-\mathbf{q}}\right] / 2=N q^{2} / 2 m$, where $N=N_{\mathrm{o}}+N_{\mathrm{c}}$ is the total number of fermions. A similar sum rule can be derived for the relative density fluctuation operator $m_{\mathbf{q}}$ [24]. In the limit when $q \rightarrow 0$ (or, more precisely, $q r_{0} \ll 1$, where $r_{0}$ is the range of actual interatomic potential),

$$
\int d \omega \omega S_{\text {rel }}(\mathbf{q}, \omega)=\frac{1}{2}\left[\left[m_{\mathbf{q}}, H\right], m_{-\mathbf{q}}\right]=\frac{N q^{2}}{2 m}-8\left\langle V_{\mathrm{oc}}\right\rangle .
$$

$\left\langle V_{\mathrm{oc}}\right\rangle$ is the coupling energy between the open and the closed channels. This is an exact relation and does not depend on the quantum state. For a true ground state, the coupling energy $\left\langle V_{\text {oc }}\right\rangle<0$, so the contribution to the sum rule is positive, while for the saddle-point solution $\left(\left\langle V_{\mathrm{oc}}\right\rangle>0\right)$, the contribution is negative. When $q=0$, the sum rule for the relative density fluctuation gives directly the coupling energy $\left\langle V_{\text {oc }}\right\rangle$, which quantifies the correlation between the open and the closed channels.

Figure 4 shows the dynamical structure factors $S_{\text {total }}(\mathbf{q}, \omega)$ and $S_{\text {rel }}(\mathbf{q}, \omega)$ for $a_{-} / a_{+}=0.8, \quad \delta=1 k_{F}^{2} \quad$ [Figs. 4(a) and 4(c)] where the Leggett mode is well defined, and $\delta=2 k_{F}^{2}$ [Figs. 4(b) and 4(d)] where the Leggett mode is within the two-particle continuum and damped [see Fig. 2(c)]. In Figs. 4(a) and 4(b), $S_{\text {total }}(\mathbf{q}, \omega)$ only features low-frequency peaks corresponding to phonon excitations while the Leggett

mode is absent. For $S_{\text {rel }}(\mathbf{q}, \omega)$, in addition to the phonon modes, there appear high-frequency peaks corresponding to well-defined [Fig. 4(c)] and damped [Fig. 4(d)] Leggett modes. Comparing Figs. 4(c) and 4(d), the spectral weight of the Leggett mode diminishes after merging into the continuum.

Investigations of the Leggett modes in multiband superconductors have already attracted intensive interests [29-34]. It is only until very recently that some evidence of its existence has been observed experimentally in multiband superconductors $\mathrm{MgB}_{2}$, by tunneling spectroscopy techniques [35], Raman spectroscopy [36], and angle-resolved photoemission spectroscopy [37]. In cold atomic systems, to observe the Leggett mode, it is necessary to engineering a coupling to the relative density operator $m_{\mathbf{q}}$. This can be achieved in the case of $\mathrm{Yb}$ atoms by using two lasers with a wave vector $\mathbf{k}_{1,2}$ and frequencies $\omega_{1,2}$ blue-detuned to the ${ }^{1} S_{0}$ to ${ }^{3} P_{1}$ transition. Due to the ac Stark shift, this generates a differential potential between the different nuclear Zeeman levels of ${ }^{1} S_{0}$. This potential is spatially modulated and takes the form [24]

$$
H^{\prime} \propto \cos (\mathbf{q} \cdot \mathbf{r}-\omega t)\left[m(\mathbf{r})+s_{\mathrm{o}}(\mathbf{r})-s_{\mathrm{c}}(\mathbf{r})\right],
$$

where $s_{\mathrm{O}, \mathrm{c}}(\mathbf{r})$ are the spin polarizations of the open and closed channels. $\mathbf{q}=\mathbf{q}_{1}-\mathbf{q}_{2}$ and $\omega=\omega_{1}-\omega_{2}$. Because of the decoupling of the spin response $s_{\mathrm{o}, \mathrm{c}}(\mathbf{r})$ from the density response [24], $H^{\prime}$ furnishes a direct probe of the Leggett modes in the relative density oscillations.

Conclusions. We have shown that for a two-component Fermi gas close to an orbital Feshbach resonance, there are in general two types of mean-field ground states that correspond to either a singlet or triplet orbital pairing symmetry, depending on the scattering parameters. The transition between the singlet and triplet states is related to the sign of the interchannel coupling energy, which we show to be related to the dynamic structure of the relative density fluctuation between the open and closed channels using exact sum rules. We also identify the emergence of the undamped Leggett mode and show that its gap vanishes at the transition point between the singlet and the triplet pairing. We calculate the dynamic structure factor corresponding to the total and relative density fluctuations and identify the signature of the Leggett mode in the relative density oscillation. To probe the Leggett mode in alkaline-earth-metal atom systems, we suggest to use Bragg spectroscopy with blue-detuned lights which can probe the relative density oscillations.

Note added. Recently, we became aware of two relevant works $[38,39]$.

Acknowledgments. We thank Hui Zhai, Bo Song, and Gyuboong Jo for very useful discussions, especially regarding the experimental feasibility of measuring the Leggett mode. This work is supported by Hong Kong Research Grants Council, General Research Fund, HKU 17306414, CRF, HKUST3/CRF/13G, and the Croucher Foundation under the Croucher Innovation Award.
[1] R. Zhang, Y. Cheng, H. Zhai, and P. Zhang, Phys. Rev. Lett. 115, 135301 (2015).
[2] G. Pagano, M. Mancini, G. Cappellini, L. Livi, C. Sias, J. Catani, M. Inguscio, and L. Fallani, Phys. Rev. Lett. 115, 265301 (2015). 
[3] M. Höfer, L. Riegger, F. Scazza, C. Hofrichter, D. R. Fernandes, M. M. Parish, J. Levinsen, I. Bloch, and S. Fölling, Phys. Rev. Lett. 115, 265302 (2015).

[4] X. Zhang, M. Bishof, S. L. Bromley, C. V. Kraus, M. S. Safronova, P. Zoller, A. M. Rey, and J. Ye, Science 345, 1467 (2014).

[5] S. Taie, R. Yamazaki, and S. Y. Takahashi, Nat. Phys. 8, 825 (2012).

[6] G. Cappellini, M. Mancini, G. Pagano, P. Lombardi, L. Livi, M. Siciliani de Cumis, P. Cancio, M. Pizzocaro, D. Calonico, F. Levi, C. Sias, J. Catani, M. Inguscio, and L. Fallani, Phys. Rev. Lett. 113, 120402 (2014).

[7] F. Scazza, C. Hofrichter, M. Höfer, P. C. De Groot, I. Bloch, and S. Fölling, Nat. Phys. 10, 779 (2014).

[8] C. Wu, J.-p. Hu, and S.-c. Zhang, Phys. Rev. Lett. 91, 186402 (2003).

[9] C. Wu, Phys. Rev. Lett. 95, 266404 (2005).

[10] H.-H. Hung, Y. Wang, and C. Wu, Phys. Rev. B 84, 054406 (2011).

[11] D. Wang, Y. Li, Z. Cai, Z. Zhou, Y. Wang, and C. Wu, Phys. Rev. Lett. 112, 156403 (2014).

[12] Z. Zhou, D. Wang, Z. Z. Y. Meng, Y. Wang, and C. Wu, Phys. Rev. B 93, 245157 (2016).

[13] A. V. Gorshkov, M. Hermele, V. Gurarie, C. Xu, P. S. Julienne, J. Ye, P. Zoller, E. Demler, M. D. Lukin, and A. M. Rey, Nat. Phys. 6, 289 (2010).

[14] M. Cazalilla, A. Ho, and M. Ueda, New J. Phys. 11, 103033 (2009).

[15] G. Pagano, M. Mancini, G. Cappellini, P. Lombardi, F. Schäfer, H. Hu, X. J. Liu, J. Catani, C. Sias, M. Inguscio, and L. Fallani, Nat. Phys. 10, 198 (2014).

[16] M. Cazalilla and A. M. Rey, Rep. Prog. Phys. 77, 124401 (2014).

[17] A. Lurio, M. Mandel, and R. Novick, Phys. Rev. 126, 1758 (1962).

[18] M. M. Boyd, T. Zelevinsky, A. D. Ludlow, S. Blatt, T. ZanonWillette, S. M. Foreman, and J. Ye, Phys. Rev. A 76, 022510 (2007).

[19] A. Bettini, Introduction to Elementary Particle Physics (Cambridge University Press, Cambridge, UK, 2014).
[20] F. Gürsey and L. A. Radicati, Phys. Rev. Lett. 13, 173 (1964).

[21] M. Iskin and C. A. R. Sá de Melo, Phys. Rev. B 72, 024512 (2005).

[22] S. N. Klimin, J. Tempere, G. Lombardi, and J. T. Devreese, Eur. Phys. J. B 88, 122 (2015).

[23] J. Xu, R. Zhang, Y. Cheng, P. Zhang, R. Qi, and H. Zhai, Phys. Rev. A 94, 033609 (2016).

[24] See Supplemental Material at http://link.aps.org/supplemental/ 10.1103/PhysRevA.95.041603 for discussions of the mean-field solutions and the collective excitations, the dynamical structure factor $S(\mathbf{q}, \omega)$ and the sum rule for the dynamical structure factor $S_{\text {rel }}(\mathbf{q}, \omega)$, and measurements of the dynamic structure factors.

[25] R. B. Diener, R. Sensarma, and M. Randeria, Phys. Rev. A 77, 023626 (2008).

[26] J. R. Engelbrecht, M. Randeria, and C. A. R. Sá de Melo, Phys. Rev. B. 55, 15153 (1997).

[27] A. J. Leggett, Prog. Theor. Phys. 36, 901 (1966).

[28] M. G. Lingham, K. Fenech, S. Hoinka, and C. J. Vale, Phys. Rev. Lett. 112, 100404 (2014).

[29] M. Ichioka, Prog. Theor. Phys. 90, 513 (1993).

[30] S. G. Sharapov, V. P. Gusynin, and H. Beck, Eur. Phys. J. B 30, 45 (2002).

[31] Y. Tanaka, Phys. Rev. Lett. 88, 017002 (2001).

[32] S. Z. Lin and X. Hu, Phys. Rev. Lett. 108, 177005 (2012).

[33] M. Marciani, L. Fanfarillo, C. Castellani, and L. Benfatto, Phys. Rev. B 88, 214508 (2013).

[34] Y. Tanaka, Supercond. Sci. Technol. 28, 034002 (2015).

[35] Ya. G. Ponomarev et al., Solid State Commun. 129, 85 (2004).

[36] G. Blumberg, A. Mialitsin, B. S. Dennis, M. V. Klein, N. D. Zhigadlo, and J. Karpinski, Phys. Rev. Lett. 99, 227002 (2007).

[37] D. Mou, R. Jiang, V. Taufour, R. Flint, S. L. Bud'ko, P. C. Canfield, J. S. Wen, Z. J. Xu, G. Gu, and A. Kaminski, Phys. Rev. B 91, 140502(R) (2015).

[38] M. Iskin, Phys. Rev. A 94, 011604 (2016).

[39] L. He, J. Wang, S. G. Peng, X. J. Liu, and H. Hu, Phys. Rev. A 94, 043624 (2016). 